\title{
Utilidad de winmedtra para conocer la prevalencia del tabaquismo en un hospital de referencia
}

\author{
Winmedtrad utility to know the smoking addiction prevalence in a reference \\ hospital
}

\section{Ranchal-Sánchez $A^{1,2,3}$, Font $\mathrm{P}^{1,2}$, López-Cabello $\mathrm{P}^{3}$, Pérula de Torres $\mathrm{LA}^{2,4}$}

1. Facultad de Medicina-Enfermería. Universidad de Córdoba. Córdoba. España.

2. IMIBIC/Hospital Regional Universitario Reina Sofía/Universidad de Córdoba. Córdoba. España.

3. Hospital Universitario «Reina Sofía». Córdoba. España.

4. Unidad Docente de Medicina Familiar y Comunitaria de Córdoba (Distrito Sanitario Córdoba y Guadalquivir). Córdoba. España.

Recibido: 07-01-15

Aceptado: 08-05-15

\author{
Correspondencia \\ Antonio Ranchal Sánchez \\ Facultad de Medicina-Enfermería \\ Avda. Menéndez Pidal s/n \\ 14001 Córdoba. España. \\ Teléfono: 699308068 \\ Correo electrónico: en 1 rasaa@uco.es
}

Resumen

Introducción: La creación de lugares de trabajo sin humo sigue siendo la medida de mayor implantación frente al tabaquismo. WinMedtra es la aplicación informática corporativa del Servicio Andaluz de Salud para la Vigilancia de la Salud.

Objetivo: Aprovechando dicha aplicación, planteamos mostrar su utilidad para calcular la prevalencia de tabaquismo en un Hospital de referencia.

Material y método: Estudio observacional a partir de los exámenes de salud realizados en 2013 $(n=1672)$. Se extrajeron los datos relativos al consumo de tabaco de WinMedtra realizándose una estadística descriptiva e inferencial (prueba "t» de Student, análisis de varianza simple y Ji-Cuadrado).

Resultados: La aplicación informática nos ha permitido obtener datos del hábito tabáquico en la población laboral estudiada. La prevalencia de sujetos fumadores activos fue de un 22,8\% (IC95\%:20,79\%$24,81 \%$ ) y el $19,8 \%$ eran ex fumadores, por lo que el $77,2 \%$ no fumaba en el periodo del estudio (IC95\%: 75,19\%-79,21\%). El consumo acumulado fue mayor en el grupo de los hombres que en el de las mujeres $(21,08 \pm 16,31$ versus $15,38 \pm 13,28$ paquetes-año) $(\mathrm{t}: 3,61, \mathrm{p}<0,001)$. Se obtuvieron diferencias significativas $(\mathrm{p}<0,001)$ al comparar la edad media de los no fumadores con respecto a la de los fumadores, $(48,33 \pm 9,82$ versus 50,55 \pm 7,62 años) y a la de exfumadores (48,33 \pm 9,82 versus 50,65 \pm 8,32 años). En relación a la categoría laboral, el mayor porcentaje de "No fumadores» pertenecía al personal residente en formación (87\%) seguido del grupo de jefaturas (75\%); siendo los auxiliares administrativos (34,7\%) y el los supervisores $(33,3 \%)$ quienes presentaban un mayor porcentaje de consumo tabáquico (Ji-cuadrado=131,23, p<0,001). 
Conclusiones: La aplicación informática puede ser útil para tener un conocimiento epidemiológico más exacto de la magnitud de conductas adictivas como el tabaquismo en centros de trabajo, obteniéndose en nuestro estudio una prevalencia de tabaquismo activo inferior a la del entorno.

Med Segur Trab (Internet) 2015; 61 (239) 172-183

Palabras clave: Prevalencia, tabaquismo, cesación tabáquica, trabajadores.

\section{Abstract}

Introduction: creating smoke-free workplaces still remains the most implemented action concerning tobacco smoking. WinMedtra is the Andalusian Health Service corporate software for the medical surveillance.

AIM: by using this application, we intend to show its utility in calculating the smoking prevalence in a reference hospital.

Material and Methods: an observational study was performed based on health examinations conducted in 2013 ( $n=1672$ ). After extracting data related with the tobacco consumption from WinMedtra, a descriptive and inferential statistics (student, simple anova and Chi-square statistical tests) were performed. We calculated arithmetic means and standard deviations for quantitative variables; and absolute and relative frequencies for qualitative variables. Confidence intervals up to $95 \%$ were calculated for the main estimates.

Results: the application allowed us to obtain smoking habits data in the interviewed working population. The smoking prevalence active subjects were 22.8\% (95\% CI: $20.79 \%-24.81 \%$ ); $19.8 \%$ were former smokers so $77.2 \%$ did not smoke during the period study (95\% CI: $75.19 \%-79.21 \%$ ). The accumulated smoking intake was higher in the group of men than in that of women $(21.08 \pm 16.31$ versus $15.38 \pm 13.28$ packs/year $)$ $(\mathrm{t}: 3.61, \mathrm{p}<0.001)$. Significant differences $(\mathrm{p}<0.001)$ were obtained by comparing the average age of nonsmokers and that of smokers, (48.33 \pm 9.82 versus $50.55 \pm 7.62$ years) and former smokers $(48.33 \pm 9.82$ versus $50.65 \pm 8.32$ years). Regarding occupational status, the highest "Non Smokers» percentage belonged to the resident workers (87\%) followed by the chief group (75\%). The group of administrative assistants (34.7\%) and the supervisors (33.3\%) had the highest «Smokers» percentage (Chi-square $=131.23, \mathrm{p}<0.001$ )

Conclusions: the software application may be useful to have an accurate epidemiological knowledge about the magnitude of addictive behavior like smoking in workplaces. We have obtained a lower smoking prevalence compared to the area in our study.

Med Segur Trab (Internet) 2015; 61 (239) 172-183

Keywords: Prevalence, Smoking Addiction, Smoking Resumption, Workers. 


\section{INTRODUCCIÓN}

Podemos considerar las actuaciones frente al humo ambiental del tabaco en los lugares de trabajo como una cuestión de salud pública. La Organización Mundial de la Salud (OMS) planteó hace años un abordaje global del control del tabaquismo ${ }^{1}$, enfermedad considerada como una epidemia ${ }^{2}$. La vigilancia del consumo del tabaco, las políticas de prevención, la protección de la población frente al humo del tabaco y la oferta de ayuda para dejar el tabaco figuran entre las seis estrategias contra el tabaquismo identificadas por la OMS, conocidas como "MPOWER" ${ }^{2,3}$. De ellas, la creación de espacios públicos y lugares de trabajo sin humo sigue siendo la medida que más y en mayor grado se ha implantado 3 .

Países como España, decidieron implementar una legislación específica frente al tabaquismo. La Ley de 2005, actualizada en 2010, potenció la puesta en marcha de programas de salud para favorecer la deshabituación tabáquica ${ }^{4}$. Previamente, Cataluña había sido la pionera en establecer una Red de Hospitales sin Humo en España ${ }^{5}$. Por su parte, Andalucía, ha desarrollado un programa integral frente al tabaquismo ${ }^{6}$, existiendo en la actualidad la "Red Andaluza de Servicios Sanitarios Libres de Humo" (RASSLH), incluida en la Red Europea de Hospitales Sin Humo (ENSH), red de la que forma parte el Hospital Universitario "Reina Sofía" (HURS) de Córdoba, desde 2013. La pertenencia a la ENSH obliga a realizar una primera y sucesivas encuestas de prevalencia ${ }^{7}$.

Por otra parte, la Ley 33/2011 General de Salud Pública establece en su artículo 3.e que: "Las actuaciones de salud pública deben evaluarse en su funcionamiento $y$ resultados.... ${ }^{8}$. Asimismo, el Real Decreto 843/2011 por el que se establecen los criterios básicos sobre la organización de recursos para desarrollar la actividad sanitaria de los servicios de prevención indica, entre las siguientes actividades sanitarias de los Servicios de Prevención (artículo 3.1), el «impulsar programas de promoción de la salud en el lugar del trabajo..." así como el "desarrollar programas de formación, información e investigación en su ámbito de trabajon".

El HURS cuenta con la especialidad de Medicina del Trabajo desde 2007, utilizando la aplicación informática WinMedtra para la gestión de la Vigilancia de la Salud desde 2011.

En este sentido, el objetivo general del artículo es mostrar la utilidad que puede tener dicha herramienta para obtener y explotar datos que nos permitan conocer, entre otros problemas de salud, la prevalencia del hábito tabáquico en un centro sanitario. Siendo un objetivo secundario describir variables socio-demográficas y del hábito tabáquico durante 2013 del personal estudiado en un Hospital de Referencia en Andalucía como es el HURS.

\section{MATERIAL Y MÉTODOS}

\section{Diseño}

Hemos realizado un estudio observacional descriptivo y de prevalencia del tabaquismo en el HURS, a partir de los datos registrados en WinMedtra ${ }^{10}$, la aplicación informática utilizada corporativamente para llevar a cabo la Vigilancia de la Salud del personal del Servicio Andaluz de Salud (SAS).

\section{Población de estudio}

La población del estudio fueron los profesionales del HURS empresa dependiente del SAS, que a su vez se integra en el Sistema Sanitario Público de Andalucía, y que emplea actualmente a unos 5000 trabajadores. De éstos estudiamos aquellos que acudieron al examen de salud y cuyos datos se registraron en WinMedtra durante 2013; por lo que se trata de una muestra de conveniencia $(\mathrm{N}=5000)$. 
El único criterio de inclusión es que fueran trabajadores en activo del HURS en el momento del examen de salud. Fueron excluidos, por tanto, el personal externo y/o de subcontratas (como es el personal de limpieza en nuestro caso). También se excluyeron aquellos que no se registraron en la aplicación informática.

\section{Procedimiento}

Una de las actividades que debe realizar obligatoriamente el personal de la Unidad de Salud Laboral es la llamada "vigilancia colectiva de la salud", la cual "no deberá ser inferior a un tercio del tiempo de trabajo" según establece la normativa en vigor ${ }^{9}$. Actuación dentro de la cual se encuadra este estudio.

El estudio ha sido aprobado por el Comité de Ética de la Investigación de Córdoba, respetando los principios fundamentales establecidos en la Declaración de Helsinki de 1964. El personal acudió a las citas programadas en función de la planificación preventiva realizada el año previo. Antes de entrar al examen de salud se le ofreció al personal un documento donde se le comunicaba explícitamente que era consciente del tratamiento informático de sus datos personales en el servicio de Vigilancia de la salud, con un espacio habilitado para la firma del trabajador. En el cuestionario autocumplimentado de salud laboral también se le preguntaba sobre hábitos de salud. Concretamente, y respecto al tabaco, que señalase si era "no fumador», "fumador» $\mathrm{O}$ «ex fumador». $Y$, en caso de fumador, el tiempo que llevaba con el hábito. Salvo los supuestos establecidos por la normativa vigente, el personal realiza el examen de salud voluntariamente, dando un consentimiento implícito para realizarlo. WinMedtra se caracteriza por el control riguroso de los datos personales de cada empleado, de acuerdo con la Ley de Protección de datos $^{10}$. La aplicación permite obtener diferentes estadísticas e índices a partir de los datos introducidos: consultas por diagnósticos, reconocimientos, actividades realizadas por el Servicio Médico, memoria anual de Vigilancia de la salud, evolución del peso y analítica del empleado, riesgo cardiovascular, índices de frecuencia, gravedad e incidencia, estadística por encuesta, y otras ${ }^{10}$. También está habilitada para crear todo tipo de cuestionarios, pudiendo obtener estadísticas de la información introducida. Estos cuestionarios pueden incluirse como parte de un protocolo específico o bien tratarlo como un programa de salud en los lugares de trabajo, como es el de la deshabituación tabáquica.

La aplicación WinMedtra contiene un apartado para el registro sistemático de datos sobre hábitos de salud. Entre otros, aquellos relativos al hábito tabáquico. Básicamente, los mismos descritos para el cuestionario autocumplimentado mencionado, además del año de inicio y abandono para quienes dejaron de fumar, el número de cigarrillos/día para el personal fumador y el ex-fumador, y otros (puros/día, pipa-Gramos/día, y si aspira o no el humo). La aplicación calcula automáticamente el número de años fumando («años hábito»). Las variables en cuestión se deben registrar sistemáticamente durante el examen de salud. Para el control del sesgo de información, dichas variables se contrastaron con las correspondientes del cuestionario general de salud laboral.

La Unidad de Coordinación de Prevención de Riesgos Laborales facilitó los datos registrados en WinMedtra mediante una base en Excel, para su tratamiento estadístico. Dicha base de datos permitió la obtención de otros relacionados, como es la variable "paquetes/año", útil para analizar el consumo acumulado de cigarrillos. Además de variables socio-demográficas como la categoría laboral. Los datos se estratificaron según género, edad y el hábito tabáquico (No fumador, fumador y exfumador).

\section{Métodos estadísticos}

Se realizó un análisis descriptivo, calculando medias aritméticas y desviaciones típicas para las variables cuantitativas, y frecuencias absolutas y relativas para las variables cualitativas. Se calcularon los intervalos de confianza al 95\% de seguridad (IC95\%). 
Para comprobar la relación entre el hábito tabáquico y las variables soicodemográficas y laborales se aplicaron las pruebas "t» de Student para datos independientes, el análisis de varianza simple y la Ji-cuadrado. Se comprobó la homogeneidad de las varianzas mediante la prueba de Levene y como contraste posthoc, se utilizó la prueba de GamesHowell.

Todos los contrastes fueron bilaterales y se consideraron significativos cuando $\mathrm{p}<0,05$.

Los datos fueron recogidos, procesados y analizados con el programa estadístico SPSS v.17.

\section{RESULTADOS}

Se obtuvo una muestra de 1672 trabajadores, con una edad media de 49,29 $\pm 9,13$ años -desviación típica- (IC95\%: 48,85-49,73 años), donde 1332 eran mujeres (79,7\%). La edad media de los hombres fue muy similar a la de las mujeres $(49,37 \pm 9,08$ versus 49,28 $\pm 9,15$ años).

La figura y la tabla 1 , respectivamente, muestran la distribución según edad y la ocupación, de quienes realizaron el examen de salud laboral en 2013.

Figura 1. Distribución según edad de la muestra estudiada

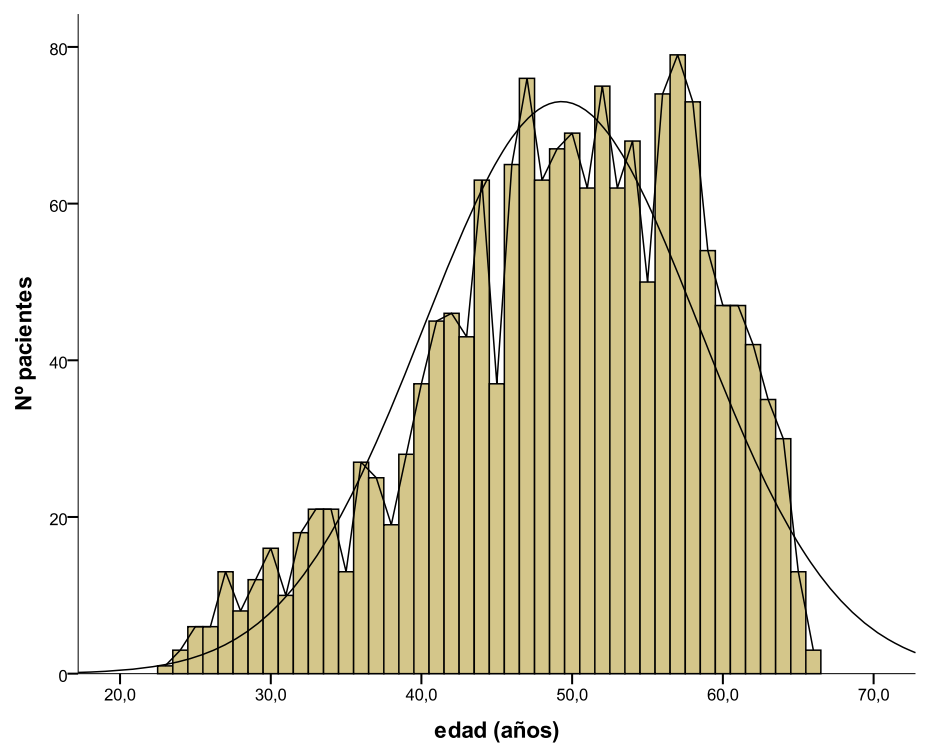


Tabla I. Distribución de la muestra estudiada según categoría laboral

\begin{tabular}{lrc}
\hline \multicolumn{1}{c}{ Categoría laboral } & $\mathbf{N}^{\circ}$ sujetos & $\%$ \\
\hline DUE/AE & 523 & 31,3 \\
\hline EIR & 46 & 2,8 \\
\hline FEA & 151 & 9 \\
\hline Supervisores & 15 & 9,0 \\
\hline Auxiliar administrativo & 392 & 23,4 \\
\hline Mantenimiento & 33 & 2 \\
\hline Hostelería & 91 & 5,4 \\
\hline Celadores & 112 & 6,7 \\
\hline Jefaturas & 16 & 1,0 \\
\hline Administración & 73 & 4,4 \\
\hline Fisioterapia & 8 & 0,5 \\
\hline Matrona & 9 & 0,5 \\
\hline Técnico especialista & 192 & 11,5 \\
\hline Otros & 11 & 0,7 \\
\hline Total & 1672 & 100 \\
\hline
\end{tabular}

DUE: Diplomado Universitario en Enfermería

AE: Auxiliar de Enfermería

FEA: Facultativo Especialista de Área

La prevalencia del hábito tabáquico fue de un $22,8 \%$ de sujetos fumadores activos (IC95\%:20,79\%-24,81\%); el 19,8\% eran ex fumadores y más de la mitad de la muestra no habían fumado nunca $(57,4 \%)$, por lo que el $77,2 \%$ no fumaba en el periodo del estudio (IC95\%:75,19\%-79,21\%).

Respecto al consumo acumulado de tabaco, por término medio fue de 16,5 $\pm 14,10$ paquetes-año para el total del grupo. Al comparar dicho consumo en relación al sexo, se obtuvieron diferencias estadísticamente significativas $(\mathrm{t}: 3,61, \mathrm{p}<0,001)$ de manera que la media de consumo acumulado fue mayor en el grupo de los hombres que en el de las mujeres $(21,08 \pm 16,31$ versus $15,38 \pm 13,28$ paquetes-año $)$.

También se obtuvieron resultados significativos (Ji-cuadrado:9,77, p=0,008) al relacionar el hábito tabáquico con el sexo, de modo que eran mujeres el 84,6\% de los fumadores, el 79,2\% de los no fumadores y el 75,2\% de los ex fumadores. Por el contrario, con respecto a los hombres, cerca de un $25 \%$ eran ex fumadores, un $20,8 \%$ no fumadores y un $15,4 \%$ fumadores (Figura 2).

Figura 2. Prevalencia de hábito tabáquico según el sexo

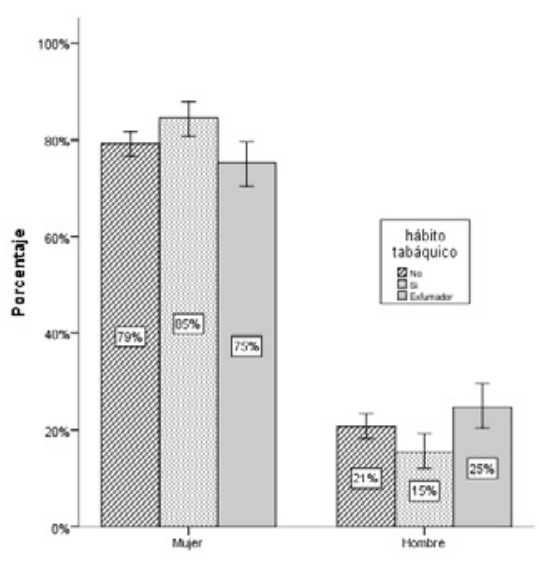


Al relacionar el hábito tabáquico con respecto a la edad de los sujetos y considerando a éstos por debajo y por encima de 50 años, no se encontraron diferencias significativas, ya que los porcentajes del hábito tabáquico por grupo de edad, fueron similares $(62,2 \%$ versus 52,2\%). Tampoco se obtuvieron resultados significativos al comparar la distribución del género en función de esos grupos de edad (79,7\% versus $79,6 \%$ de mujeres, respectivamente).

Con respecto al promedio de edad de los sujetos en relación al hábito tabáquico, se obtuvieron diferencias significativas ( $\mathrm{t}: 12,80, \mathrm{p}<0,001)$ al comparar la edad media de los no fumadores con respecto a la de los fumadores, (48,33 $\pm 9,82$ versus $50,55 \pm 7,62$ años) y a la de exfumadores (48,33 $\pm 9,82$ versus $50,65 \pm 8,32$ años); las edades medias de los fumadores y exfumadores no difirieron entre sí (Figura 3 ).

Figura 3. Edad media (IC95\%) de los pacientes según hábito tabáquico

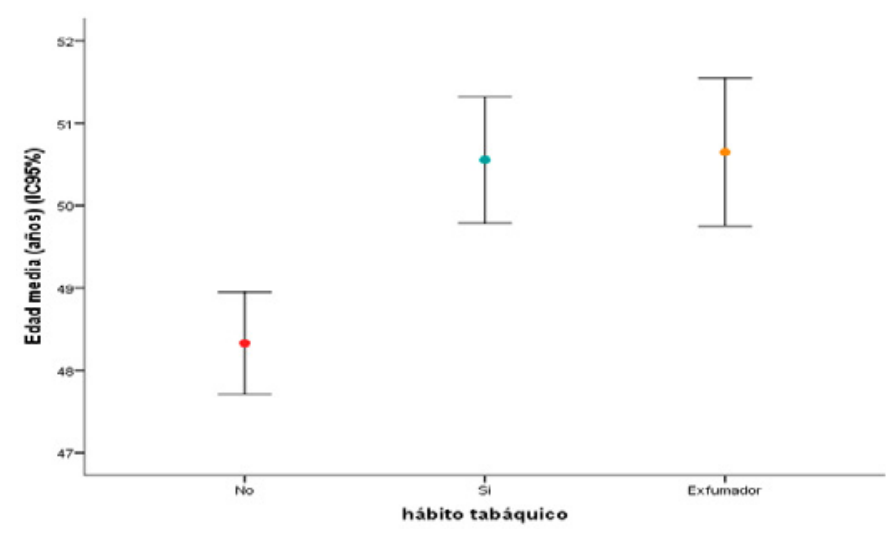

Por último, en cuanto a la distribución de los sujetos en relación a la categoría laboral, el mayor porcentaje de la muestra de "No fumadores» pertenecía al personal residente en formación (EIR), con un $87 \%$, seguido del grupo de jefaturas (75\%); siendo el grupo de auxiliares administrativos (34,7\%) y el de supervisores (33,3\%) quienes presentaban un mayor porcentaje de fumadores ( $\mathrm{Ji}$-cuadrado= 131,23; $\mathrm{p}<0,001$ ).

\section{DISCUSIÓN}

El objetivo principal del estudio era mostrar la utilidad de la aplicación para explotar y analizar datos epidemiológicos, centrándonos en los obtenidos en relación al hábito tabáquico para describir la prevalencia de tabaquismo en la población estudiada. Mostramos que, además de ser un software para realizar los exámenes de salud de la Vigilancia individual, se puede rentabilizar obteniendo datos grupales o colectivos con utilidad para los responsables de prevención de riesgos laborales, de cara a implementar o ajustar sus programas o protocolos de actuación.

WinMedtra es un instrumento potencialmente útil para facilitar la vigilancia colectiva de la salud, vigilancia a la que obliga la normativa ${ }^{9}$, echándose en falta publicaciones que demuestren su realización en la práctica. La aplicación permite obtener estadísticas detalladas de las actividades realizadas en cada servicio de prevención. En este sentido, pensamos que el artículo muestra la utilidad práctica que puede tener dicha herramienta en la obtención de la prevalencia del hábito tabáquico de un centro sanitario y del perfil sociodemográfico y laboral del consumidor de esta sustancia tóxica. Dato de obligado cumplimiento para las empresas que participan en la RASSLH, y por ende, en la ENSH, como indicador de las actuaciones realizadas.

Los resultados de nuestro estudio mostraban una prevalencia del hábito tabáquico de un $22,8 \%$ de sujetos fumadores activos en 2013.La Encuesta Andaluza de Salud ${ }^{11}$ (EAS) 2011-2012 indica una prevalencia de población andaluza fumadora mayor de 16 años de 
un $30,9 \%$ en 2011 (26,8\% en los hombres y $25,2 \%$ en las mujeres). Dicha EAS $^{11}$ muestra que un 30,1\% fumaba a diario en Córdoba, ciudad donde se ubica el Hospital analizado. Estudios realizados en estudiantes universitarios andaluces muestran también una mayor prevalencia de tabaquismo ${ }^{12}$ (26,3\% en 2011) que la encontrada en nuestro estudio, pese a la "influencia de las leyes anti tabaco". Por su parte, Vicente-Herrero et $\mathrm{l}^{13}$, indican, en su estudio de 2008, una cifra de fumadores del 35\% en trabajadores del sector de Correos, cifra que, según ellos mismos indican, está por encima del 30\% que indicaba la Encuesta Nacional de Salud en $2006^{13}$, años después de implantada la Ley frente al tabaquismo ${ }^{4}$. Datos estos a favor de la idea de que la legislación, por sí sola, no es suficiente para reducir la prevalencia en la población fumadora y, concretamente, en la trabajadora.

El tabaquismo, enfermedad considerada como una epidemia ${ }^{2}$, requiere de una vigilancia y abordaje global para su control ${ }^{1}$. Abordaje que debe encuadrarse en un programa de promoción de la salud en el lugar del trabajo9 , más allá de ser una cuestión de obligado cumplimiento legal ${ }^{4}$ y de constituir un riesgo de incendio cuando ésta se incumple. Por otra parte, sabemos que es la causa principal de muchas enfermedades pulmonares, con un coste económico para la EPOC $^{14}$ de hasta un 3,6\% del Producto Interior Bruto.

La vigilancia del consumo del tabaco, las políticas de prevención, la protección de la población frente al humo del tabaco y la oferta de ayuda para dejar el tabaco figuran entre las seis estrategias para el control del tabaco identificadas por la OMS, conocidas como "MPOWER»" ${ }^{2,3}$. En este sentido, el HURS comenzó en 2008 un programa integral frente al tabaquismo, con medidas de sensibilización, señalización, información y cumplimiento de la normativa, lideradas y dirigidas por la Dirección de Servicios Generales. Programa, encuadrado en el Plan Integral del Tabaquismo de Andalucía ${ }^{6}$, que se ha mantenido en el tiempo e incorporado en la actual RASSLH, habiendo obtenido el HURS en 2014 la medalla de plata del ENSH. Los expertos indican que aquellos hospitales con mayor tiempo de adhesión a la Red protegen mejor el ambiente y procuran lugares de trabajo más saludables ${ }^{7}$, por lo que las acciones realizadas para favorecer la deshabituación tabáquica en el hospital han debido influir en el 19,8\% de ex fumadores que indican nuestros resultados. Deshabituación realizada en la consulta específica, a cargo de un especialista en Medicina del Trabajo. Consulta que se ofrece al personal fumador del Hospital. Dell'Olmo et al. ${ }^{15}$ concluyen que los «Médicos del trabajo pueden jugar un papel importante en la deshabituación tabáquica mediante el consejo breve durante la vigilancia de la salud y su participación en programas para la promoción salud en el trabajo". Refuerza esta idea el hecho de que un estudio realizado en el mismo HURS y publicado en $1996^{16}$, indicaba una prevalencia de consumo de tabaco del $42 \%$, si bien se necesitan más estudios centrados en los facultativos, población objeto de dicho trabajo $^{16}$ para conocer el impacto en dicho colectivo. Otros autores indican que la intervención en tabaquismo realizadas por médicos de hospital son efectivas ${ }^{17}$. Está asimismo demostrado que la implementación de programas para la deshabituación tabáquica en empresas implica beneficios tanto en salud como económicos desde su puesta en marcha ${ }^{18}$. La intervención frente al tabaquismo es positiva para reducir el impacto que tienen tanto las patologías pulmonares ${ }^{19,20}$, como otras prevalentes por la mortalidad y morbilidad que conlleva esta enfermedad adictiva crónica ${ }^{20}$ en nuestro entorno.

Por otra parte, los datos registrados en la aplicación WinMedtra permiten analizar la estructura socio-demográfica y laboral de la muestra estudiada, objetivo secundario del trabajo. En nuestro caso, podríamos hablar de una población laboral envejecida (figura 1), con mayoría de trabajadoras y con predominio del personal de enfermería en el colectivo sanitario (tabla 1). Datos, estos últimos, parecidos, con los expresados en la página web del hospital ${ }^{21}$, según la cual, el 52,30\% pertenecen a la dirección de enfermería (enfermeras, auxiliares de enfermería, matronas, fisioterapeutas, personal técnico...) $(51,40 \%$ en la muestra) y el $20,30 \%$ a la dirección médica (11,78\% en la muestra), siendo el $27,30 \%$ no sanitarios $^{21}$ (36,82\% en la muestra). Un $72 \%$ de la plantilla está integrado por mujeres ${ }^{21}$, siendo el dato obtenido en nuestra muestra de un $79,7 \%$ de mujeres. El análisis de la 
estructura poblacional laboral debería ser el punto de partida a la hora de implementar programas de salud. Por ejemplo, el relativo a actuaciones para el "empoderamiento" de la salud de las personas mayores en los lugares de trabajo, colectivo que debiera ser preferente en las actuaciones de seguridad y salud en el trabajo ${ }^{22}$.

Respecto al género, prevalece la población laboral fumadora femenina frente a la masculina (figura 2), dato a tomar con cautela puesto que predominan las trabajadoras, no habiendo podido ser estandarizados los resultados para tomar en consideración esta variable. Sin embargo, la misma EAS ${ }^{11}$ indica que el tabaquismo femenino se incrementó en dos puntos porcentuales entre 2007 y 2011. Hecho que orienta hacia la importancia de dirigir acciones específicas atendiendo a la variable de género. Por ejemplo, durante el embarazo, situación que, pese a ser idónea para abandonar el tabaquismo, tiene una prevalencia media de un $21,6 \%{ }^{23}$. Por el contrario, el consumo acumulado (índice paquetes-año), obtuvo un promedio superior en los hombres $(21,07)$ frente a las mujeres $(15,37)$ en nuestro estudio.

Con relación a la edad, el personal no fumador tiene un promedio de edad menor que el fumador y el ex fumador (figura 3). Profesionales jóvenes como los EIR obtuvieron un porcentaje inferior de tabaquismo que otros colectivos.

Las conclusiones derivadas de los resultados obtenidos mediante el sistema de información empleado presentan algunas limitaciones. Una de ellas es el sesgo de selección que se ha podido producir al contar con una muestra de conveniencia, es decir, los sujetos fueron incluidos utilizando un procedimiento de muestreo no probabilístico, y ello dificulta y genera incertidumbre sobre su representatividad con respecto a la población de estudio. La muestra del estudio la compone el personal que, habiendo acudido voluntariamente a vigilancia de la salud, sus datos son registrados y codificados adecuadamente. La aplicación informática permite analizar solo aquello que se registra. Esto es, "lo que no se registra y/o no se codifica no existe epidemiológicamente», limitando la representatividad de la muestra. El registro de los datos del hábito tabáquico en la aplicación WinMedtra no lleva más de 30 segundos. Cuantos más datos del hábito tabáquico se registren, más amplia es la muestra, y, por tanto, más extrapolable a la población de estudio. La codificación sistemática de las variables analizadas permitiría obtener una visión bastante aproximada a la realidad de la prevalencia del hábito tabáquico en el mundo laboral y el seguimiento de la evolución del tabaquismo en dicho ámbito.

La plantilla del HURS cuenta actualmente con más de 5000 profesionales, por lo que la muestra obtenida $(n=1672)$, es cuantitativamente grande $(33,4 \%)$, y ello permitiría pensar que podría ser representativa del conjunto de la población de estudio aunque cabe pensar que la magnitud del problema estudiado también pueda estar sobreestimado, pues, es plausible que sean aquellos trabajadores más preocupados por su salud, buscando ayuda o consejo médico para tratar de dejar de fumar, los que acudan a este programa de vigilancia de la salud. Pensemos acerca de la utilidad que puede tener la aplicación en hospitales pequeños, donde más que de muestra estaríamos hablando de población de estudio. Así como la posibilidad para comparar tanto entre empresas del mismo sector, como inter-sectores que utilicen la misma aplicación informática.

El estudio puede adolecer también del sesgo del "trabajador sano», puesto que la muestra la constituye personal que, voluntariamente, accedió al examen de salud. No obstante, existe literatura realizada en otros colectivos de trabajadores (sector de Correos) donde también se obtienen los datos mediante la captación durante la realización de exámenes de la vigilancia periódica de la salud ${ }^{13}$. Asimismo, existen estudios europeos de prevalencia tabáquica realizados en médicos de Hospital, cuya muestra procede de los datos recogidos en los exámenes de salud de los trabajadores ${ }^{15}$.

Por otra parte, la falta de una tasa de participación según categoría laboral, es una limitación del trabajo. Existen categorías, como la correspondiente a los FEA, con baja frecuentación a los exámenes de vigilancia de la salud (tabla 1). Puede deducirse que un $11,78 \%$ de este colectivo realizó el examen de salud en 2013 (9\% excluyendo a los EIR, 
cuyo examen de salud es obligatorio ${ }^{24}$ ), cuando un $20,30 \%$ del personal corresponde a la dirección médica del HURS ${ }^{21}$. En esta línea, una propuesta sería que el programa informático incorporara la capacidad para establecer una «llamada» cuando la frecuentación de una categoría laboral no alcanza el «mínimo deseable». También la incorporación de un módulo de explotación estadística descriptiva que permitiese realizar, automáticamente, el desglose por categorías y la explotación de los datos por el propio Médico de Vigilancia de la Salud. Sería, por otra parte deseable que, al igual que el programa informático cumple todas las normativas del Proyecto Científico “CARPE-DIEM" ${ }^{25}$, se adaptase también a lo establecido en el Proceso Asistencial Integrado "Atención a las Personas Fumadoras" ${ }^{26}$; al menos en lo que a la Historia Clínica homónima se refiere. También limita el trabajo el hecho de que no permita vincular datos de los hábitos de salud del personal externo, como es el de la limpieza en nuestro centro. Por lo que sería conveniente algún módulo que permitiera incorporar, desde el punto de vista estadístico, la explotación de los datos obtenidos desde el servicio de prevención correspondiente.

Para terminar, respecto a la variable profesión, debemos llamar la atención sobre el hecho de que enfermería sea el personal sanitario con mayor porcentaje de fumadoras observado. Dato coincidente con otros estudios de centros pertenecientes a la $\mathrm{ENSH}^{7}$. La literatura indica que fuman más las enfermeras de atención especializada que las de primaria $^{27}$. Bien es cierto que enfermería es el personal predominante en número en el Hospital. Precisamente por eso debe ser el colectivo de atención preferente. Siendo, además el personal que más tiempo dispensa al paciente, por lo que su función como profesionales modélicas resulta fundamental. Estudios previos ya demostraban que las políticas antitabaco limitan el impacto del comportamiento de los fumadores, y sugerían que estrategias futuras deberían concentrarse en la actuación sobre el personal de enfermería tanto en las restricciones como en el apoyo para cambiar su comportamiento ${ }^{28}$. Especialmente en determinados Servicios/Unidades como es el de Salud Mental ${ }^{29}$, y en el colectivo de supervisoras, mediante actuaciones específicas enmarcadas en un plan integral antitabaco. Políticas que debieran favorecer la inclusión de la formación en diagnóstico y tratamiento del tabaquismo en el currículo de los estudios de enfermería, tanto a nivel de grado como de postgrado. Especialmente en los Hospitales Universitarios, como el HURS, acreditado en calidad en prevención de riesgos laborales (OHSAS 180012007), que forman también a personal de enfermería especialista residente. Expertas en la materia plantean incluso la presencia de enfermeras especializadas en deshabituación tabáquica ${ }^{7}$. En este sentido, la especialista en enfermería del trabajo tiene un perfil idóneo, al incluir su formación la promoción de la salud laboral ${ }^{30}$.

En conclusión la prevalencia global de tabaquismo activo en trabajadores del HURS puede considerarse inferior a la del entorno. Prevalencia obtenida a partir de los exámenes de salud realizados mediante WinMedtra durante 2013, demostrando la aplicabilidad práctica de la aplicación, puesto que el estudio de la prevalencia es unos de los ítems exigidos para pertenecer a la RASSLH. No obstante, sugerimos ulteriores estudios que mejoren las limitaciones del trabajo así como otros que se centren en el personal sanitario y, específicamente, en el facultativo.

\section{AGRADECIMIENTOS}

A don José Luis Gómez de Hita, Director de la Dirección de Servicios Generales del HURS, por el liderazgo en el programa de actuación frente al tabaquismo.

A la Unidad de Coordinación de Prevención de Riesgos Laborales (Dirección General de Profesionales) del Servicio Andaluz de Salud, por facilitar la base de datos. 


\section{REFERENCIAS BIBLIOGRÁFICAS}

1. Framework Convention on Tobacco Control: development of an evidence based global public health treaty. Brit Med J. 2003; 327:147-7.

2. WHO report on the global tobacco epidemic, 2009: Implementing smoke-free environments. Disponible en: http://www.who.int/tobacco/mpower/2009/gtcr_download/en/

3. WHO report on the global tobacco epidemic, 2013. Enforcing bans on tobacco advertising, promotion and sponsorship. Disponible en:http://www.who.int/tobacco/global_report/2013/en/

4. Ley 42/2010, de 30 de diciembre, por la que se modifica la Ley 28/2005, de 26 de diciembre, de medidas sanitarias frente al tabaquismo y reguladora de la venta, el suministro, el consumo y la publicidad de los productos del tabaco. BOE núm. 318, de 31 de diciembre de 2010.

5. Méndez E. Las políticas de espacios libres de humo: la Red de Hospitales sin Humo en Cataluña. Prev Tab. 2002;4:93-4.

6. Consejería de Salud. Junta de Andalucía. Plan Integral de Tabaquismo de Andalucía 2005-2010, 1. reimpresión 2007.

7. Martínez C y García M. Evaluación del grado de implantación de las intervenciones para el control del tabaquismo en la Red Catalana de Hospitales sin Humo. Enferm Clin. 2007; 17(4):177-85.

8. Ley 33/2011, de 4 de octubre, General de Salud Pública. BOE núm. 240, de 5 de octubre de 2011.

9. RD 843/2011, de 17 de junio, por el que se establecen los criterios básicos sobre la organización de recursos para desarrollar la actividad sanitaria de los servicios de prevención.

10. Aplicación WinMedtra. Características. Disponible en: http://www.atmedtra.es

11. Consejería de Igualdad, Salud y Políticas Sociales. Junta de Andalucía. Encuesta Andaluza de Salud 20112012. Muestra en Adultos. Disponible en: http://www.juntadeandalucia.es/salud/sites/csalud/contenidos/ Informacion_General/c_3_c_1_vida_sana/vigilancia_de_la_salud/encuesta?perfil=org

12. Rojas J, Soto JG, García A, Cabrera C. Prevalencia del tabaquismo en estudiantes del Campus Universitario de Jerez. Influencia de las leyes anti tabaco. Aten Primaria 2013.

13. Vicente-Herrero MT, López-González AA, Pont-Martínez P, et al. Situación actual del tabaquismo entre los trabajadores de correos-España tras la Ley 28/2005. Med Segur Trab 2008; 54 (213): 87-95.

14. Briceño DM, et al. La carga económica de la EPOC. Análisis de los costos a nivel internacional. Neumol Cir Torax 2011; 70 (2):118-126.

15. Dell'Olmo M, Abbritti G, Folleti I, et al. Reduction of tobacco smoking among physicians of a hospital in central Italy. G Ital Med Lav Ergon 2012; 34 (3):453-5.

16. Mengual P, Pérula LA, Redondo J, Roldán A, Prada A, Martínez J, Ruiz R. Evolución del consumo y actitud ante el tabaco de los médicos del hospital regional Reina Sofía de Córdoba. Gac Sanit. 1996; 10: 18-24.

17. Raupach T, Merker J, Hasenfuss G, et al. Knowledge gaps about smoking cessation in hospitalized patients and their doctors. Eur J Cardiovasc Prev Rehabil. 2011; 18 (2) 334-41.

18. Diaz S, Martín I. Impacto presupuestario de un programa de deshabituación tabáquico en empresas. Med Segur Trab (internet) 2009; 55 (216): 46-55.

19. European Lung Foundation \& European Respiratory Society (ERS). La Salud Pulmonar en Europa, hechos y cifras. 2014.

20. Jiménez-Ruiz C, Fagerström KO. Tratado de Tabaquismo. 2. ${ }^{\text {a }}$ Ed. Madrid: Ergon; 2007.

21. Distribución en porcentajes del personal del Hospital Universitario "Reina Sofía». Disponible en: http:// www.juntadeandalucia.es/servicioandaluzdesalud/hrs3/index.php?id=profesionales

22. Communication from the Commission to the European Parliament, the Council, the European Economic and Social Committee and the Committee of the Regions on an EU Strategic Framework on Health and Safety at Work 2014-2020. European Commission, Brussels, 6.6.2014 COM (2014) 332 final.

23. Mateos-Vilchez P, Aranda-Regules JM, Díaz-Alonso G, et al. Prevalencia del tabaquismo durante el embarazo y factores asociados en Andalucía, 2007-2012. Rev Esp Salud Pública. 2014;88:369-381.

24. Orden SSI/1674/2014, de 10 de septiembre, por la que se aprueba la oferta de plazas y la convocatoria de pruebas selectivas 2014 para el acceso en el año 2015, a plazas de formación sanitaria especializada para Médicos, Farmacéuticos, Enfermeros y otros graduados/ licenciados universitarios del ámbito de la Psicología, la Química, la Biología y la Física. BOE núm. 226, de 17 de septiembre de 2014.

25. Programa CARPE DIEM. Campaña de Actuación y Registro Precoz de la EPOC Dirigida a Empresas. Disponible en http://www.aeemt.com/contenidos/Anuncio_Actividades/PROYECTO\%20CARPE\%20 DIEM\%202013/ProyectoCarpeDiem.pdf 
26. Autoría múltiple. Proceso Asistencial Integrado: Atención a las Personas Fumadoras. 1. ${ }^{\mathrm{a}}$ Ed. Sevilla: Junta de Andalucía, Consejería de Salud; 2008.

27. Fernández- Ruiz ML y Sánchez-Bayle. Evolución de la prevalencia de tabaquismo entre las médicas y enfermeras de la Comunidad de Madrid. Gac Sanit. 2003; 17 (1):5-10.

28. Strobl J y Latter S. Qualified nurse smoker's attitudes towards a hospital smoking ban and its influence on their smoking behavior. J Adv Nurs. 1998; 27 (1): 179-88.

29. Bloor RN, Meeson L, Crome IB. The effects of a non-smoking policy behaviour and attitudes in a psychiatric hospital. J Psychiatr Ment Health Nurs. 2006; 13 (2):188-96.

30. Orden SAS/1348/2009, de 6 de mayo, por el que se aprueba y publica el programa formativo de la especialidad de Enfermería del Trabajo. BOE núm. 129, de 28 de mayo de 2009.

|| 